\title{
Factores de riesgo para anormalidades citológicas del cuello uterino en pacientes atendidas en los establecimientos de salud, de la provincia de Huaraz, 2013
}

\author{
Risk factors for cervical cytological abnormalities in patients served in health \\ facilities of the province of Huaraz, 2013 \\ Marcelo Arotoma Oré1 ${ }^{1}$ Olga Cayra Sahuanay ${ }^{1}$, Enid Arotoma Nuñez ${ }^{1}$, \\ Rosa Ríos Salinas ${ }^{1}$ y Ángel Mendoza López ${ }^{1}$
}

\section{RESUMEN}

Este estudio determina los factores de riesgo para anormalidades citológicas del cuello uterino en pacientes atendidas en los establecimientos de salud: Hospital Víctor Ramos Guardia, Centros de Salud: Huarupampa, Nicrupampa y Monterrey, de la Provincia de Huaraz, 2013. Hipótesis de la investigación: Los factores de riesgo que presentan las pacientes atendidas en los establecimientos de salud de la provincia de Huaraz, influyen significativamente para la aparición de las anormalidades citológicas del cuello uterino; en comparación con las mujeres que no presentaron dichos factores de riesgo. Metodológicamente el estudio descriptivo, observacional, transversal y prospectivo; epidemiológico de casos y controles. La muestra estuvo constituida por 65 mujeres con anormalidades citológicas del cuello uterino (casos) y 65 sin anormalidades citológicas (controles). Los resultados fueron procesados en el Programa Estadístico SPSS versión 20.0, y la prueba del Odds Ratio (O.R.) con intervalo de confianza al 95\% y un nivel de significación de $5 \%(\mathrm{p}<0,05)$. Según esto las anormalidades citológicas de cérvix identificadas fueron: 35,4\% (23) ASCUS, LEI-BG: 27,7\% (18), LEI-AG: 18,5\% (12) y PVH: 18,4\% (12). Los factores de riesgo para anormalidades citológicas del cuello uterino fueron: edad de inicio de vida sexual $\leq$ de 18 años, primer parto $\leq$ de 18 años, gran multípara $\geq$ de 5 partos, parejas sexuales $\geq$ de 2 , sin estudio citológico y exposición a método anticonceptivo oral $\geq 5$ años.

Palabras clave: citología; histología; zona de transformación.

\section{ABSTRACT}

This research determines risk factors for cervical cytological abnormalities in patients treated in health facilities: Victor Ramos Guardia Hospital, health centres: Huarupampa, Nicrupampa and Monterrey in the province of Huaraz, 2013. Research hypothesis: The risk factors presented by the patients treated at health facilities in the province of Huaraz to significantly influence the development of cervical cytological abnormalities; compared with women who did not have these risk factors: Descriptive, observational, transversal study; epidemiological case-control; it shows 65 women with cervical cytological abnormalities (cases) and 65 without cytological abnormalities (controls). The results were processed in the Statistical Program SPSS version 20.0, and proof Odds

Universidad Nacional «Santiago Antúnez de Mayolo». Huaraz, Perú. 
Ratio (OR) with confidence interval of $95 \%$ and a significance level of $5 \%(\mathrm{p}<0,05)$. Accordingto that, the cytological cervical abnormalities identified were: 35,4\% ASCUS, LEI-BG: 27,7\% (18), LEI-AG: 18,5\% (12) y HPV: 18,4\% (12). Risk factors for cervical cytological abnormalities were: age of onset of sexual life $\leq 18$ years, first bith $\leq 18, \geq 5$ large multiparous births $\geq 2$ sexual partners without cytology and exposure to oral contraceptive $\geq 5$ years.

Keywords: cytology; histology; transformation zone.

\section{ICHIKLLACHAW}

Kay musyapakuy uryayqa kayraykurmi rurakashqa: llapan huwakturkunapa mantsayninta situluhikakunapa mana alli kayninta, utirinu kunkan qishyaqkunachaw, kay rurakashqa «Víctor Ramos Guardia» hatun hampikuna wayichawmi, hinaman wakin ichik hampikuna wayikunachaw: Warupampa, Nikrupampa, Muntiriy, kaykuna Waraspa markanchawmi kayan, kaykuna 2013 watachaw rurakashqa. Kay musyapakuypa iputisisninkuna kashqa: llapan huwakturkuna imanawshi kaykan llapan qishyaqkunapa kaasuyninkuna, tsay Waraspa markanchaw, hampikuna wayinkunachaw, kaykuna imanawmi alliq awqan situluhiya mana alli kayninman utirinupa kunkanchaw, wakin warmikuna manam kay mantsakay huwakturkuna kayaapuntsu. Matiryalwan mitudukunaqa: yachakuy niymi, rikachakuymi, ñawpamanwan llapanman aywaq kaasukunawan kuntrulkunam, 65 warmikunapa situluhiyankuna mana alli kaynin kaq manam kayaapuntsu. Kay risultadukuna rurakashqa istadistiku SPSS hutiyuq prugramachawmi 20.0 birsiyunchaw, Odds Ratiopa (O.R.) chanintsayninwan, intirbalupa kunhuwiyansanwan 95\%, alli kaq nibilnin 5\% $(\mathrm{p}<0,05)$. Yarquyninkuna: situluhiyapa sirbixnin mana alli kayninkuna kashqa 35,4\% (23) ASCUS, LEIBG: 27,7\% (18), LEI. AG: $18,5 \%$ (12) hina PVH: 18,4\% (12). Kaykunapa uchukllachaw ushayninkuna kaykunam kashqa: llapan huwakturkuna mantsaynin utirinu kunkanpa situluhiyapa mana alli kayninkuna kayashqa: siksupa qallanan kawaynin $\leq 18$ watayuqpa, punta kaq wachay $\leq 18$ watayuqpa, hatun multiipara $\geq 5$ wachaypa, siksuwal mahakuna $\leq 2 \mathrm{pa}$, mana situluhiku yachakuyninwan antikunsiptibu ural mitudun niyninwan $\geq 5$ watayuq.

Pushaq shimikuna: situluhiya; istuluhiya; transhurmasiyunpa kaqninman.

\section{INTRODUCCIÓN}

El propósito de la investigación fue identificar en la población femenina de la provincia de Huaraz los factores de riesgo para las anormalidades citológicas del cuello uterino, ya que en la actualidad la citología cervical anormal y el cáncer del cuello uterino son frecuentes entre las mujeres del mundo, reportándose el cáncer del cuello uterino como el tercero en frecuencia en mujeres (530,000 nuevos casos, $13,6 \%$ del total); el 85\% de los casos registrados se produce en los países en desarrollo (453,000 casos); las tasas más altas están en las regiones del Este Africano, específicamente en Zimbabwe y Uganda, con tasas de incidencia estandarizada de 47,3 y 45,8 casos por 100,000 mujeres. (Ferlay et al., 2010). Mientras que las tasas de incidencia más bajas se han encontrado en Israel (población no judía) con 2,4 x 100,00 y en Egipto con 2,1 por 100,000 (Ferlay et al., 2010). 
En cuanto a la mortalidad se estima 275,000 en el año 2008 a nivel mundial, convirtiendo el cáncer del cuello uterino en la séptima causa de muerte; las tasas de mortalidad más altas se encuentran en el continente africano y en el sudeste asiático 21,7 y 13,7 x 100,000 mujeres respectivamente; y las tasas de mortalidad más bajas se encuentran en la región del medio oriente y norte de África (3,0 x 100,000). (Ferlay, et al., 2010). En el Perú,según MINSA (2011) la tasa de incidencia nacional de anormalidades citológicas es de 28,8 x 100,000 mujeres, y la tasa de mortalidad es de 11,3 por 100,000. La ciudad de Trujillo tiene la tasa más alta 43,9; seguida por Arequipa con 35,2 y Lima, la tasa más baja con 19, 6 (ob. cit.). El examen de frotis de Papanicolaou (PAP) y su lectura por médico patólogo, tiene décadas de experiencia en su uso; bajo costo, alta especificidad puede revelar una lesión pre cancerosa, la displasia cervical, así como cáncer in situ o un cáncer invasivo en sus primeras fases; el tratamiento temprano de estas lesiones es sumamente eficaz; mientras que la enfermedad en sus fases más avanzadas puede ser mortal a pesar del intento de tratamiento avanzado (Miller, 2010).

Hay muchos factores de riesgo para el cáncer cervical así como: mujeres mayores de 25 años, inicio temprano de relaciones sexuales antes de los 19 años, multiparidad, embarazo temprano (antes de 18 años), infección cervical por virus del papiloma humano (VPH), antecedente de infección de transmisión sexual, promiscuidad sexual, tabaquismo, nunca haberse practicado estudio citológico de cérvix, exposición a elevados niveles de estrógeno y antecedentes familiares de cáncer del cuello uterino; estos factores son considerados como de calidad superior para las anormalidades citológicas a largo plazo (Miller, 2010).

El trabajo de investigación es considerado porque tiene relevancia social; ya que se fundamenta en el beneficio que tendrán las mujeres en la práctica del examen PAP rutinario, parte del cuidado de su salud, especialmente las mujeres con factor de riesgo. Pues, en ellas se ha detectado las anormalidades citológicas del cuello uterino. Además tiene utilidad metodológica porque los resultados del presente estudio servirán a las autoridades del Ministerio de Salud de Áncash, para la toma de decisiones en la práctica de la prevención y promoción de la salud y luego se logre preservar la salud sexual y reproductiva de las mujeres.

El objetivo general planteado fue: determinar los factores de riesgo para anormalidades citológicas del cuello uterino en mujeres atendidas en los establecimientos de salud: Hospital Víctor Ramos Guardia, Centros de Salud: Huarupampa, Nicrupampa y Monterrey en el año 2013; y como objetivos específicos: Identificar el tipo de anormalidades citológicas del cuello uterino, teniendo en cuenta el Sistema de Bethesda: Células escamosas de significado indeterminado (ASCUS). Lesión Escamoso Intraepitelial de Bajo Grado (LEI-BG), incluye: Papiloma Virus Humano (PVH), Lesión Escamoso Intraepitelial de Alto Grado (LEI-AG) y explorar los principales factores de riesgo asociados al surgimiento de anormalidades citológicas del cuello uterino en la población en estudio.

\section{MATERIALES Y MÉTODOS}

Tipo de estudio descriptivo, observacional, transversal y prospectivo; epidemiológico de casos y controles; la muestra, 65 mujeres que se han sometido a la toma de la muestra de PAP, como parte del cuidado de su salud, previa firma de consentimiento infor- 
mado, las muestras en una lámina previamente fijada fueron remitidas al laboratorio, luego procesadas por el médico Patólogo y los resultados fueron informados utilizando un formulario validado por el Ministerio de Salud del Perú: el sistema BETHESDA (Tipo de informe estandarizado, para resultados de estudios de muestra PAP, tales como: PVH, LEI-BG, LEI-AG, cáncer in situ, etc.). La información fue procesada en el Programa Estadístico SPSS versión 20.0; se utilizó la prueba Odss Ratio (OR) con intervalo de confianza AL 95\% y un nivel de significancia de 5\% $(p<0,05)$.

\section{RESULTADOS}

Se presenta los resultados obtenidos, según los objetivos del trabajo de investigación titulado: Anormalidades citológicas del cuello uterino en pacientes atendidas en los establecimientos de Salud, de la provincia de Huaraz, 2013.

Tabla 1. Tipo de anormalidades citológicas del cuello uterino, según sistema Bethesda y grupo etáreo atendidas en los establecimientos de salud de la provincia de Huaraz, 2013

\begin{tabular}{lcccccccccr}
\hline $\begin{array}{l}\text { Anormalidad } \\
\text { citológica }\end{array}$ & $\mathrm{f}$ & $\%$ & $15-19$ & $\%$ & $20-29$ & $\%$ & $30-39$ & $\%$ & $40-49$ & $\%$ \\
\hline PVH & 12 & 18,4 & 0 &, 0 & 3 & 4,6 & 7 & 10,7 & 2 & 3,0 \\
ASCUS & 23 & 35,4 & 1 & 1,5 & 9 & 13,8 & 6 & 9,2 & 7 & 10,7 \\
LEI-BG & 18 & 27,7 & 0 &, 0 & 1 & 1,5 & 11 & 16,9 & 6 & 9,2 \\
LEI-AG & 12 & 18,5 & 0 &, 0 & 1 & 1,5 & 4 & 6,1 & 7 & 10,7 \\
Total & 65 & 100 & 1 & 1,5 & 14 & 21,5 & 28 & 43,0 & 22 & 33,8 \\
\hline
\end{tabular}

Las anormalidades citológicas de mayor prevalencia en la población estudiada fueron: ASCUS, $1,6 \%$ (23) de mujeres, seguido de LEI-BG 1,3\% (18), LEI-AG y PVH 0,8\% (12); por grupo etáreo fueron: ASCUS 35,4\% en mujeres de 20-29 años, LEI-BG 27,7\% (18) entre 30-39 años; mientras que PVH y LEI-AG (18,4\%) en grupo etáreo de 30-39 años.

Tabla 2. Edad de inicio de vida sexual de mujeres con anormalidades citológicas del cuello uterino atendidas en los establecimientos de salud de la provincia de Huaraz, 2013.

\begin{tabular}{cccccccc}
\cline { 2 - 7 } & \multicolumn{2}{c}{ Casos } & \multicolumn{2}{l}{ Controles } & \multicolumn{2}{c}{ Total } \\
\hline $\begin{array}{c}\text { Edad de inicio de } \\
\text { vida sexual }\end{array}$ & $\mathrm{n}=65$ & \multicolumn{2}{c}{$\mathrm{n}=65$} \\
$<18$ años & 51 & 57,9 & 37 & 42,1 & 88 & 100 \\
$\geq 18$ años & 14 & 33,3 & 28 & 66,6 & 42 & 100 \\
Total & 65 & 50, & 65 & 50,0 & 130 & 100 \\
OR: 2,757 & IC: $1,278-5,946$ & & $\mathrm{p}=0,015$ & \\
\hline
\end{tabular}

Se observa 57,9\% (51) mujeres del grupo, casos que iniciaron su vida sexual antes de los 18 años de edad; el grupo de control fue 42,1\%(37). Realizando el análisis estadístico se obtuvo valor de OR: 2,757 con un IC: $1,27-5,9$, no contienen a la unidad y $\mathrm{p}<0,05$, variable considerada significativa, es factor de riesgo para las anormalidades citológicas de cérvix. 
Tabla 3. Edad del primer parto con anormalidades citológicas del cuello uterino atendidas en los establecimientos de salud de la provincia de Huaraz, 2013

\begin{tabular}{ccccccc}
\cline { 2 - 7 } & \multicolumn{2}{c}{ Casos } & \multicolumn{2}{c}{ Controles } & \multicolumn{2}{c}{ Total } \\
\hline Edad del primer & \multicolumn{2}{c}{$\mathrm{n}=65$} & \multicolumn{2}{c}{$\mathrm{n}=65$} \\
parto & $\mathrm{f}$ & $\%$ & $\mathrm{f}$ & $\%$ & $\mathrm{f}$ & $\%$ \\
$\leq 18$ años & 40 & 59,7 & 27 & 40,3 & 67 & 100 \\
$\geq 18$ años & 25 & 39,6 & 38 & 60,4 & 63 & 100 \\
Total & 65 & 50,0 & 65 & 50,0 & 130 & 100 \\
OR: 2,170 & \multicolumn{3}{c}{ IC: $1,020-4,650$} & $\mathrm{p}=0,044$ \\
\hline
\end{tabular}

El 59,7\% (40) de los casos tuvo su primer parto antes de los 18 años; en el grupo de control fue 40,3\% (27). El análisis estadístico obtuvo valor de OR: 2,170 un IC: 1,04,6 , que no contiene a la unidad y valor de $\mathrm{p}<0,05$, siendo esta variable significativa, se considera riesgo para las anormalidades citológicas del cuello uterino.

Tabla 4. Número de partos de mujeres con anormalidades citológicas atendidas en los establecimientos de salud de la provincia de Huaraz, 2013

\begin{tabular}{ccccccc} 
& \multicolumn{2}{c}{ Casos } & \multicolumn{2}{c}{ Controles } & \multicolumn{2}{c}{ Total } \\
\hline Número de partos & \multicolumn{2}{c}{$\mathrm{n}=65$} & \multicolumn{2}{c}{$\mathrm{n}=65$} & & \\
Primípara (1 parto) & 7 & 100,0 & 0 &, 0 & 7 & 100 \\
Multípara (2-5 partos) & 6 & 40,0 & 9 & 7,0 & 15 & 100 \\
Gran multípara $(\geq 5$ partos) & 52 & 48,15 & 56 & 51,8 & 108 & 100 \\
Total & 65 & 0,0 & 65 & 50,0 & 130 & 100 \\
Chi $^{2}=7,748$ & & & $\mathrm{p}=0,021$ & & \\
\hline
\end{tabular}

El 48,15\% (52) de casos fue de grandes multíparas ( $\geq$ de 5 partos) y del grupo de control 51,8\% (56). Realizando el análisis estadístico se obtuvo el valor de $\mathrm{p}<0,05$, variable considerada significativa, es factor de riesgo para las anormalidades citológicas del cuello uterino.

Tabla 5. Número de parejas sexuales de mujeres con anormalidades citológicas atendidas en los establecimientos de salud de la provincia de Huaraz, 2013

\begin{tabular}{cccccccc} 
& \multicolumn{3}{c}{ Casos } & \multicolumn{3}{c}{ Controles } & \multicolumn{2}{c}{ Total } \\
\hline Número de parejas & \multicolumn{2}{c}{$\mathrm{n}=65$} & \multicolumn{2}{c}{$\mathrm{n}=65$} \\
sexuales & $\mathrm{f}$ & $\%$ & $\mathrm{~F}$ & $\%$ & $\mathrm{f}$ & $\%$ \\
Una pareja & 15 & 41,6 & 21 & 58,4 & 36 & 100 \\
$\geq$ de 2 parejas & 50 & 53,2 & 44 & 46,8 & 94 & 100 \\
Total & 65 & 50,0 & 65 & 50,0 & 130 & 100 \\
OR:3,667 & \multicolumn{3}{c}{ IC: $1,588-8,466$} & $\mathrm{p}=0,003$ \\
\hline
\end{tabular}


El 53,2\% (50) del grupo de casos tuvieron mayor o igual número de dos parejas sexuales; y en el grupo de control fue el 46,8\% (44). Realizando el análisis estadístico se obtuvo el valor de OR: 3,667 y un IC: 1,5-8,4, no conteniendo a la unidad y p<0,05; variable considerada significativa, es factor de riesgo para las anormalidades citológicas del cuello uterino.

Tabla 6. Tiempo de exposición al anticonceptivo oral en mujeres con anormalidades citológicas atendidas en los establecimientos de salud de la provincia de Huaraz, 2013

\begin{tabular}{ccccccc} 
& \multicolumn{2}{c}{ Casos } & \multicolumn{2}{c}{ Controles } & \multicolumn{2}{c}{ Total } \\
\hline Tiempo uso & \multicolumn{2}{c}{$\mathrm{n}=65$} & \multicolumn{2}{c}{$\mathrm{n}=65$} \\
MAC oral & $\mathrm{f}$ & $\%$ & $\mathrm{f}$ & $\%$ & $\mathrm{f}$ & $\%$ \\
$>$ de 5 años & 60 & 48,4 & 64 & 51,6 & 124 & 100 \\
$\leq$ de 5 años & 5 & 83,3 & 1 & 16,6 & 6 & 100 \\
Total & 65 & 50,0 & 65 & 50,0 & 130 & 100 \\
OR:1,103 & \multicolumn{3}{c}{ IC: $1,201-6,003$} & $\mathrm{p}=0,0311$ \\
\hline
\end{tabular}

En cuanto al tiempo de exposición al método anticonceptivo oral el 48,4\% (60) de los casos utilizó dicho anticonceptivo por un tiempo $\geq$ de 5 años, mientras que en el grupo de control 51,6\%(64) de mujeres. Realizando el análisis estadístico se obtuvo el valor de OR: 1,103 y un IC: 1,201-6,003 que no contiene a la unidad y p>0,05; siendo esta variable estadísticamente significativa, es factor de riesgo para las anormalidades citológicas del cuello uterino.

Tabla 7. Número de papanicolaou de mujeres con anormalidades citológicas atendidas en los establecimientos de salud de la provincia de Huaraz, 2013

\begin{tabular}{ccccccc}
\cline { 2 - 7 } & \multicolumn{2}{c}{ Casos } & \multicolumn{2}{c}{ Controles } & \multicolumn{2}{c}{ Total } \\
\hline Número de PAPs & \multicolumn{2}{c}{$\mathrm{n}=65$} & \multicolumn{2}{c}{$\mathrm{n}=65$} \\
tomados & $\mathrm{f}$ & $\%$ & $\mathrm{f}$ & $\%$ & $\mathrm{f}$ & $\%$ \\
Nunca & 33 & 56,9 & 25 & 43,1 & 58 & 100 \\
Una vez & 11 & 45,8 & 13 & 54,2 & 24 & 100 \\
Más de dos veces & 21 & 43,8 & 27 & 56,3 & 48 & 100 \\
Total & 65 & 50,0 & 65 & 50,0 & 130 & 100 \\
OR:2,707 & \multicolumn{3}{c}{ IC: $1,311-8,015$} & $\mathrm{p}=0,0364$ \\
\hline
\end{tabular}

Se observa que el 56,9\% (33) de los casos afirmó nunca haberse practicado dicho examen y el grupo de control 43,1(25) de mujeres. Previo análisis estadístico se obtuvo valor de OR: 2,707, IC: 1,311-8,015, no conteniendo la unidad y $\mathrm{p}<0,05$; siendo esta variable estadísticamente significativa, se considera como factor de riesgo para anormalidades citológicas del cuello uterino. 


\section{DISCUSIÓN}

Entre los hallazgos de anormalidades citológicas del cuello uterino identificadas, prevalece ASCUS, en 35,4\% de mujeres; existe coincidencia con los reportados por Alterio et al. (2007) quien demostró esta patología en 22,2\% de su muestra a diferencia de Magallanes (2009) que los observo en 53,8\% de su muestra. Se identificó a la LEI-BG, en $27,7 \%$ de la muestra; resultado cercano de Meza et al. (2005) y Alterio et al. (2007) en $23,20 \%$ y $22,2 \%$ de pacientes. $18,5 \%$ de mujeres presentó LEI-AG, difiriendo con los resultados de Peláez, (2010), ya que solo 3,1\% de su muestra presentó dicha anormalidad citológica. 18,4\% de mujeres tuvo PVH; en la actualidad este virus es considerado como causa primaria para el cáncer del cuello uterino, transmitido sexualmente como los del serotipo 16 y 18 (OMS, 2010); existe coincidencia con los resultados de Luna, (2010) 8\% de pacientes presentó dicha patología, a diferencia de los hallazgos de Cabrera (2011); pues 64,8 \% de las mujeres estuvieron expuestas al PVH.

Se determinó los factores de riesgo que conllevan a las anormalidades citológicas del cuello uterino como: Inicio de vida sexual activa antes de los 18 años 57,9\% de mujeres del grupo de casos y 42,1\% en el grupo de control; siendo esta variable estadísticamente significativa, es importante resaltar la coincidencia con los resultados de las investigaciones de Meza (2005), Alterio et al. (2007), Luna (2010), Magallanes (2009), Lazo (2011) y Cabrera (2011) quienes en similares porcentajes identificaron al inicio de vida sexual activa antes de los 18 años como un factor de riesgo para el desarrollo de anormalidades citológicas. Además, los expertos de la Organización Mundial de la Salud (OMS), sostienen que la zona de transformación del epitelio cervical es la más proliferativa durante la pubertad y la adolescencia. Por lo tanto, el coito a una edad temprana, puede modificar y transformar esta zona; además existe la posibilidad de exponerse a infecciones de transmisión sexual, lo que conlleva en un futuro a desarrollar infecciones crónicas y con esto, elevar el riesgo de contraer anormalidades citológicas de cérvix y por consiguiente cáncer al cuello uterino (Miller, 2010).

Se evidenció que 59,7\% de mujeres del grupo de casos tuvieron su primer parto siendo menor de 18 años, mientras que en el grupo de control fue de 40,3\%; variable estadísticamente significativa; similares resultados obtuvieron: Magallanes (2009) y Cabrera (2011) que determinaron en 50\% y 55\% de su muestra que las mujeres tuvieron su primer parto antes de 18 años; considerando a esta variable como factor determinante para anormalidades citológicas, como lo sostenido por los investigadores de la OMS; pues, mujeres cuyo antecedente de parto es menor a los 18 años, presentaron mayor porcentaje de patologías de cérvix (OMS, 2010).

Sobre paridad, 48,1\% de casos presentó anormalidades citológicas del cuello uterino, correspondiendo a mujeres con número mayor de cinco partos, variable considerada estadísticamente significativa; es importante resaltar la coincidencia con los resultados de Peláez (2010) y Magallanes (2009) que comparten datos de 40,0\% y 34,6\% de su muestra en que las mujeres tuvieron más de cinco hijos y presentaban anormalidades citológicas de cérvix; una afectación inmunológica repetida influye en la aparición de 
una infección con facilidad, en especial con el PVH; además de los efectos traumáticos que se producen a nivel del cuello uterino en cada parto (OMS, 2010).

Se identificó 53,2\% del grupo de casos que tuvieron mayor número o igual a dos parejas sexuales; encontrándose una similitud con el resultado del investigador Alterio et al. (2007), quien determinó que el 44,4\% de su muestra presentaó anormalidades citológicas de cérvix porque tuvieron más de dos parejas sexuales. El tener mayor número de parejas sexuales representa un factor de riesgo para anormalidades citológicas del cuello uterino, esto se relaciona directamente con una mayor exposición al PVH (Miller, 2010).

El 48,4\% del grupo de casos estuvo expuesto a métodos anticonceptivos orales por tiempo mayor a cinco años; este resultado es congruente con la literatura médica de la historia natural de las anormalidades citológicas, ya que indica que a mayor tiempo de exposición del método anticonceptivo oral, pueden ser considerados casos como iniciadores de dicha patología (Ibíd.).

El 56,9\% de mujeres del grupo de casos, afirmaron no haberse realizado examen de PAP; este resultado difiere significativamente con los obtenidos por Cabrera (2011) pues 20\% de su muestra presentó anormalidades citológicas de cérvix. La literatura médica sostiene al grupo de pacientes con vida sexual activa y que nunca se han sometido a estudio citológico de cérvix como los que tienen mayor probabilidad de padecer anormalidades citológicas de cérvix, lo que contrasta con nuestro resultado (Ibid.).

\section{CONCLUSIONES}

El tipo de anormalidades citológicas del cuello uterino que, siguiendo en orden de prevalencia fueron: Células escamosas de significado indeterminado detectadas en 35,4\% de mujeres del grupo etáreo de 20-29 años, seguido de LEI-BG en 27,7\% de 30-39 años, LEI-AG en 18,5\% de 40-49 años y PVH en 18,4\% de mujeres cuyas edades correspondieron al rango de 30-39 años. No se logró detectar ningún caso de cáncer al cuello uterino.

Los principales factores de riesgo identificados para las anormalidades del cuello uterino fueron: edad de inicio de vida sexual $\leq$ a 18 años, la edad del primer parto $\leq$ de 18 años, la gran multiparidad $\geq$ de 5 partos, el número de parejas sexuales $\geq$ de 2 , nunca haberse realizado estudio citológico de cérvix y exposición al método anticonceptivo oral combinado $\geq$ de 5 años con valores de $\mathrm{p}<0,05$. Considerados estadísticamente significativos, con dichos resultados se comprobó la hipótesis planteada.

\section{AGRADECIMIENTOS}

A la Universidad Nacional «Santiago Antúnez de Mayolo» por el apoyo económico en la realización de la presente investigación. 


\section{REFERENCIAS BIBLIOGRÁFICAS}

Alterio, Gianfranco; Mendoza, Indira y Mendoza, Rosalía. 2007. Hallazgos citológicos y factores de riesgo para patología pre invasora de cuello uterino. Venezuela. <http:/ /www. respyn.uanl.mx/viii/3/articulos/hallazgos_citologicos.htm $>$ [Consulta: 02-01$13]$.

Cabrera, José. 2011. Distribución de los factores de riesgo en el cáncer de cuello uterino en pacientes del Hospital Nacional Sergio E. Bernales. 2006-2011. Universidad Nacional Mayor de San Marcos. <http://cybertesis.unmsm.edu.pe/bitstream/cybertesis/452/1/ cabrera_aj.pdf $>$ [Consulta: 04-04-13].

Ferlay, Jacques y otros. 2010. Cancer Incidence and Mortality Worldwide. GLOBOCAN 2010. <http://globocan.iarc.fr> [Consulta: 02-02-13].

Lazo, Erick y Aguilar, Frank. 2011. Relación entre factores de riesgo reproductivo de mujeres com citologia cervical uterina alterada. Hospital II-2 Tarapoto. <http:/ / fisi.unsm.edu. pe/spunsm///archivos_proyectox/archivo_82_Binder1.pdf $>$ [Consulta: 1202-13].

Luna, María. 2010. Factores de riesgo asociados al padecimiento de cáncer de cérvix en usuarias de la Unidad de Salud de Ilobasco, Departamento de Cabañas. Universidad El Salvador. <http://www.umoar.edu.sv/tesis/Ingenieria\%20Industrial/salud $\% 20$ publica $\% 20$ maestria.pdf $>$ [Consulta: 09-03-13].

Magallanes, Jesús; Villanueva, Maritza y Mejía, Alberto. 2009. Factores de riesgo reproductivo de mujeres con citología cérvico uterina alterada. Hospital Regional de Loreto. <http://www.unapiquitos.edu.pe/oficinas/iiunap/archivos/2009/medicina/ ArticuloJesusMagallanes.pdf.> [Consulta: 10-10-13].

Meza, Shopia y otros. 2005. Prevalencia de Hallazgos Citológicos del cuello uterino y los factores de riesgo asociados en la población femenina. Universidad Centro Occidental Lisandro Alvarado. <http://bibmed.ucla.edu.ve/DB/bmucla/edocs/textocompleto/ TPWP470F74200 5.pdf.> [Consulta: 12-01-13].

Miller, Anthony. 2010. Programas de Detección del Cáncer cérvico uterino; Directrices de gestión, Ginebra: OMS.

Ministerio de Salud del Perú. 2011. Epidemiología. Lima: Oficina General de Epidemiología, Publicación anual.

Peláez, Luzmila y Rivas, Javier. 2010. Prevalencia de anomalías citológicas en frotis cervical y factores asociados en citología realizadas en el Hospital Vicente Corral Moscoso. Ecuador. $<$ http://dspace.ucuenca.edu.ec/bitstream/123456789/3711/1/MED173.pdf> [Consulta: 12-10-13].

Fecha de recepción: 30 de febrero de 2015

Fecha de aceptación: 15 de mayo de 2015 
||Marcelo Arotoma, Olga Cayra, Enid Arotoma, Rosa Ríos y Ángel Mendoza

\section{Correspondencia}

Marcelo Arotoma Oré

ido_1954_1@hotmail.com 